\title{
Archival materials
}

\section{Bodleian Library, Oxford}

Add Ms. D/114

Ash Ms. 826

Carte Ms. 80

Clarendon Ms. 22-23

English History Ms. C 53

Nalson Ms. 13

Rawlinson Ms. B 48

Tanner Ms. 62-63, 66
Oxford Siege Book

War Accounts

March 1643 - January 1644

Diary, 1642-1644

London in Arms Displayed

\section{British Library}

Additional MS 968

Additional MS 5494

Additional MS 7532

Additional MS 10114

Additional MS 18777-18779

Additional MS 18781-18782

Additional MS 22619

Additional MS 27962

Additional MS 31116

Additional MS 34253

Additional MS 34315
London Regiments by Symonds

Accounts and Papers Relating to

Sequestered Estates, 1642-1648

The Rights of the People of England

Papers of John Harington

Diary of Walter Yonge

Reports of sermons in London, 1642-4

Collection of original papers

Salvetti correspondence

Diary of Lawrence Whitacre

Correspondence relating to the civil war

Committee of Safety receipts 


\begin{tabular}{|c|c|}
\hline Additional MS 37343 & Whitelocke's Annals vol. iii \\
\hline Additional MS 40630 & Cassiobury Papers \\
\hline Additional MS 40883 & Diary of Nehemiah Wallington \\
\hline Additional MS 71534 & Henry Marten Papers \\
\hline Additional MS 72435 & $\begin{array}{l}\text { Trumbull Papers [Walter Strickland's } \\
\text { correspondence] }\end{array}$ \\
\hline $\begin{array}{r}\text { Additional MSS } \\
39940-39942\end{array}$ & Notes on sermons \\
\hline BL Egerton MS 1048 & London Petitions from December 1642 \\
\hline $\begin{array}{l}\text { BL Egerton MS 2643, } \\
2654,2647\end{array}$ & Barrington Papers \\
\hline BL Harleian MS 162-166 & Diary of Sir Simonds D'Ewes \\
\hline BL Harley MS 479 & Diary of John More \\
\hline BL Sloane MS 654 & Letter correspondence \\
\hline BL Sloane MS 922 & Letter book of Nehemiah Wallington \\
\hline BL Sloane MS 1465 & Petitions and letters \\
\hline BL Sloane MS 1457 & A Memoriall of Gods Judgment \\
\hline BL Sloane MS 1467 & Petitions and Speeches in Parliament \\
\hline BL Stowe MS 142 & miscellaneous historical letters \\
\hline
\end{tabular}

\section{Camden Local Studies and Archives Centre}

VOL P/GF/M/1

St. Giles in the Fields, Vestry Minutes, 1618-1719

VOL P/GF/M/4

St. Giles in the Fields, Churchwardens' Accounts, 1640-1694

\section{Clothworkers' Company, London}

Orders of Courts, 1639-1649

\section{Drapers’ Company, London}

Court Minutes and Records, 1640-1667 


\section{Essex Record Office}

$\mathrm{T} / \mathrm{B}, 211$

\section{Goldsmiths' Company, London}

Book 02-W

MS 223
Company Minutes, 1642-1645

Strelley's Charity Book, 1603-1790

\section{Huntington Library, San Marino}

Ellesmere MSS

Papers of John Egerton, earl of Bridgewater

Hastings MSS

Huntington MSS

Stowe MSS

\section{John Harvard Library, Southwark}

St. George the Martyr Churchwardens' Accounts, 1621-170

St. Mary Newington Churchwarden’ Accounts, 1632-1734

\section{Lambeth Palace Library, Southwark}

MS 679

MS 703

MS 930

MS 932

\section{Leathersellers' Company, London}

Court Minutes, 1608- 


\section{London Guildhall Library}

MS 182/2

MS 913

MS 1207/3-4

MS 2208/1

MS 2881/6-7

MS 2883/1

MS 3043/1

MS 3293/1-2

MS 3297/1

MS 4329/4-5

MS 4655/5-6

MS 5177/4

MS 5204/3

MS 5220/2

MS 5257/5-6

MS 5385

MS 5445/3-7

MS 5570/3

MS 5603/1

MS 5606/1

MS 5667

MS 6112/1

MS 6122/2

MS 6152/2

MS 6153/1-2

MS 6649/1
Court Minute Book of the Paviors' Company

Court Minute Book of the Society of Tacklehouse and Ticket Porters

Court Minute Books Armourers' and Braisers' Company

Court Minute Book of the Plumbers' Company

Court Minute Books of the Blacksmiths' Company

Wardens' Account Book of the Blacksmiths' Company

Court Minute Book of the Tylers' and Bricklayers' Company

Court Minute Books of the Turners' Company

Wardens' Account Book of the Turners' Company

Court Minute Books of the Carpenters' Company

Court Minute Books of the Weavers' Company

Court Minute Book of the Bakers' Company

Court Minute Book of the Brown-Bakers' Company

Court Minute Book of the Gunmakers' Company

Court Minute Books of the Barber-Surgeons' Company

Court Minute Book of the Saddlers' Company

Court Minute Books of the Brewers' Company

Court Minute Book of the Fishmongers' Company

Court Minute Book of the Coopers' Company

Wardens' Account Book of the Coopers' Company

Court Minute Book of the Painter-Stainers' Company

Court Minute Book of the Curriers' Company

Court Minute Book of the Plaisterers' Company

Wardens' Account Book of the Tallow Chandlers' Company

Court Minute Books of the Tallow Chandlers' Company

Court Minute Book of the Inholders' Company 


\begin{tabular}{|c|c|}
\hline MS 7090/4-5 & Court Minute Books of the Pewterers' Company \\
\hline MS 7151/1 & Court Minute Book of the Cutlers' Company \\
\hline MS 7158/1 & Wardens' Account Book of the Cutlers' Company \\
\hline MS 7353/14-15 & Court Minute Books of the Cordwainers' Company \\
\hline MS 8041/1 & $\begin{array}{l}\text { Wardens' Account Book of the Joiners' and Ceilers' } \\
\text { Company }\end{array}$ \\
\hline MS 9485/1 & $\begin{array}{l}\text { Court Minute Book of the Wax Chandlers' } \\
\text { Company }\end{array}$ \\
\hline MS 11588/4 & Court Minute Book of the Grocers' Company \\
\hline MS $14346 / 2$ & Wardens' Account Book of the Curriers' Company \\
\hline MS 15201/1 & Court Minute Book of the Vintners' Company \\
\hline MS 15842/1 & Court Minute Book of the Haberdashers' Company \\
\hline MS $16967 / 4$ & Court Minute Book of the Ironmongers' Company \\
\hline MS $30708 / 3$ & Court Minute Book of the Skinners' Company \\
\hline MS 34017/5 & $\begin{array}{l}\text { Court Minute Book of the Merchant Taylors' } \\
\text { Company }\end{array}$ \\
\hline
\end{tabular}

\section{London Metropolitan Archives}

\begin{tabular}{|c|c|}
\hline CLC/180/MS07415/001 & $\begin{array}{l}\text { Dutch Church at Austin Friars, } \\
\text { Afkondingen [Proclamation] } \\
\text { book, 1643-1752 }\end{array}$ \\
\hline CLC/180/MS07399 & $\begin{array}{l}\text { Copy out-letter book, comprising } \\
\text { copies of letters from the } \\
\text { Consistory, mainly to other } \\
\text { Dutch reformed congregations }\end{array}$ \\
\hline CLC/270/MS03342 & London in armes displayed \\
\hline COL/AD/01/041 & $\begin{array}{l}\text { Court of Common Council } \\
\text { Letter Book QQ }\end{array}$ \\
\hline COL/CA/01/01/059 & $\begin{array}{l}\text { Court of Aldermen Repertory 55, } \\
3 \text { November } 1640 \text { - } 13 \text { August } \\
1642\end{array}$ \\
\hline COL/CA/01/01/060 & $\begin{array}{l}\text { Court of Aldermen Repertory 56, } \\
18 \text { August } 1642-24 \text { October } \\
1643\end{array}$ \\
\hline
\end{tabular}


COL/CA/01/01/061

COL/CHD/CT/01/004

COL/CHD/CT/01/005

COL/CHD/MN/03/005

COL/CHD/MN/01/004

COL/CHD/MN/01/006

COL/CHD/MN/02/015

COL/CN/01/01/001

Ms. 824/1

P69/ALH1/G/01/001

P69/ALH1/H/05/001

P69/ALH4/B/001/MS04049/001

P69/ALH6/B/001/MS04957/001

P69/ALH6/B/008/MS04956/003/001

P69/ALH7/B/001/MS00819/001

P69/ALH7/B/013/MS00818/001
Court of Aldermen Repertory 57, 31 October 1643 - 28 October 1645

City's Cash Accounts, 1641-3

City's Cash Accounts, 1644-6

Warrant of the Militia

Committee of the City of London to the Committee of Arrears, 24 April 1646

Maimed soldiers and sailors: Account book, 1665-79

Military and Naval Pensions, 1661-79

Miscellaneous papers, 1643-90

Common Hall, Corporation of London Minutes, November 1642 - October 1646

All Hallows the Less, Vestry Minutes, 1644-1830

All Hallows Barking by the Tower, Vestry Minutes, 1629-69

All Hallows Barking by the Tower, Churchwardens' Accounts, 1628-66

All Hallows Lombard Street, Vestry Minutes, 1618-53

All Hallows Staining Mark Lane, Vestry Minutes, 1574-1655

All Hallows Staining Mark Lane, Churchwardens' Accounts, 1645-79

All Hallows the Great, Vestry Minutes, 1574-1684

All Hallows the Great, Churchwardens' Accounts, 1616-1708 
P69/ALH8/B/013/MS00823/001

P69/ALB/B/001/MS01264/001

P69/ALB/B/003/MS07673/002

P69/ALP/B/001/MS01431/002

P69/ALP/B/006/MS01432/004

P69/TRI3/B/004/MS04835/001

P69/AND3/B/001/MS01278/001

P69/AND3/B/003/MS01279/003

P69/AND1/B/009/MS02088/001

P69/ANA/B/010/MS00587/001

P69/ANL/B/004/MS01046/001

P69/BAT1/B/001/MS04384/001

P69/BAT1/B/001/MS04384/002

P69/BAT1/B/006/MS04383/001
All Hallows the Less, Churchwardens' Accounts, 1630-51

St. Alban Wood Street, Vestry Minutes, 1583-1676

St. Alban Wood Street, Churchwardens' Accounts, 1637-75

St. Alphage London Wall, Vestry Minutes, 1608-1711

St. Alphage London Wall, Churchwardens' Accounts, 1631-77

Holy Trinity the Less, Churchwardens' Accounts, 1582-1662

St. Andrew Hubbard, Vestry Minutes, 1600-78

St. Andrew Hubbard, Churchwardens' Accounts, 1621-1712

St. Andrew-by-the-Wardrobe, Churchwardens' Accounts, 1570-1688

St. Anne and Saint Agnes, Churchwardens' Accounts, 1636-63

St. Antholin Budge Row, Churchwardens' Accounts, 1574-1708

St. Bartholomew-by-theExchange, Vestry Minutes, 1567-1643

St. Bartholomew-by-theExchange, Vestry Minutes, 1643-76

St. Bartholomew-by-theExchange, Churchwardens' Accounts, 1598-1698 
P69/BEN1/B/005/MS01303/001

P69/BEN2/B/001/MS04214/001 pt.2

P69/BEN3/B/007/MS00878/001

P69/BEN3/B/001/MS00877/001

P69/BOT1/B/001/MS01453/001

P69/BOT2/B/001/MS09236

P69/BOT2/B/012/MS09235/002/002

P69/BOT3/B/007/MS00942/001

P69/BOT4/B/001/MS04526/001

P69/BOT4/B/008/MS04524/002

P69/BRI/B/016/MS06552/001

P69/CRI/B/007/MS04423/001

P69/CRI/B/001/MS04425/001

P69/CLE/B/007/MS00977/001

P69/CLE/B/001/MS00978/001

P69/DUN2/B/011/MS02968/003
St. Benet Fink, Churchwardens Accounts, 1610-99

St. Benet Gracechurch Vestry Minutes, 1607-1758

St. Benet Paul's Wharf, Churchwardens' Accounts, 1605-57

St. Benet Paul's Wharf Vestry Minutes, 1579-1674

St. Botolph Aldersgate, Vestry Minutes, 1601-52

St. Botolph Aldgate, Vestry Minutes, 1583?-1708

St. Botolph Aldgate, Churchwardens' Accounts, 1586-1691

St. Botolph Billingsgate, Churchwardens' Accounts, 1603-74

St. Botolph-without-Bishopsgate, Vestry Minutes, 1616-90

St. Botolph-without-Bishopsgate, Churchwardens' Accounts, 1632-62

St. Bride Fleet Street, Churchwardens' Accounts, 1639-78

St. Christopher le Stocks, Churchwardens' Accounts, 1575-1660

St. Christopher le Stocks, Vestry Minutes, 1593-1731

St. Clement Eastcheap, Churchwardens' Accounts, 1636-1740

St. Clement Eastcheap, Vestry Minutes, 1640-1759

St. Dunstan-in-the-West Churchwardens' Accounts, 1628-44 
P69/DUN2/B/001/MS03016/001

P69/ETH/B/006/MS04241/001

P69/GEO/B/001/MS00952/001

P69/GEO/B/005/MS00951/001

P69/GRE/B/001/MS01336/001

P69/HEL/B/004/MS06836

P69/JS2/B/001/MS04813/001

P69/JS2/B/005/MS04810/002

P69/JNB/B/006/MS00577/001

P69/JNZ/B/014/MS00590/001

P69/KAT1/B/011/MS01124/001

P69/KAT2/B/001/MS01196/001

P69/LAW1/B/001/MS02590/001

P69/LAW1/B/008/MS02593/002

P69/LAW2/B/010/MS03907/001
St. Dunstan-in-the-West Vestry Minutes, 1588-1663

St. Ethelburga Bishopsgate, Churchwardens' Accounts, 1569-1681

St. George Botolph Lane and St. Botolph Billingsgate Vestry Minutes, 1600-85

St. George Botolph Lane Churchwardens Accounts, 1590-1676

St. Gregory by St. Paul, Vestry Minutes, 1642-1701

St. Helen Bishopsgate Churchwardens' Accounts, 1565-1654

St. James Garlickhithe, Vestry Minutes, 1615-93

St. James Garlickhithe, Churchwardens' Accounts, 1627-99

St. John the Baptist Walbrook, Churchwardens' Accounts, 1595-1679

St. John Zachary, Churchwardens' Accounts, 1591-1682

St. Katherine Coleman Street, Churchwardens' Accounts, 1610-71

St. Katherine Cree, Vestry Minutes, 1639-1718

St. Lawrence Jewry, Vestry Minutes, 1556-1670

St. Lawrence Jewry, Churchwardens' Accounts, 1640-98

St. Laurence Pountney, Churchwardens' Accounts, 1530-1681 
P69/LAW2/B/001/MS03908/001

P69/MAG/B/018/MS01179/001

P69/MGT1/B/001/MS04352/001

P69/MGT3/B/001/MS01175/001

P69/MGT3/B/014/MS01176/001

P69/MGT4/B/001/MS04571/001

P69/MGT4/B/004/MS04570/002

P69/MRY1/B/006/MS03891/001

P69/MRY2/B/001/MS03570/002

P69/MRY2/B/005/MS03556/002

P69/MRY3/B/010/MS06574

P69/MRY4/B/001/MS01240/001

P69/MRY8/B/001/MS00064
St. Laurence Pountney, Vestry Minutes, 1614-73

St. Magnus the Martyr, Churchwardens' Accounts, 1638-1734

St. Margaret Lothbury, Vestry Minutes, 1571/2-1677

St. Margaret New Fish Street, Vestry Minutes, 1583-1675

St. Margaret New Fish Street, Churchwardens' Accounts, 1576-1678

St. Margaret Pattens, Vestry Minutes and Memoranda Book, 1640-83

St. Margaret Pattens, Churchwardens' Accounts, 1558-1653

St. Martin Ludgate, Vestry Minutes, 1576-1715

St. Martin Orgar, Vestry Minutes, 1469-1707

St. Martin Outwich, Churchwardens' Accounts, 1632-1743

St. Mary Abchurch, Churchwardens' Accounts, 1629-92

St. Mary Aldermanbury, Vestry Minutes, 1610-1763

St. Mary Aldermanbury, Churchwardens' Accounts, 1631-77

St. Mary Aldermary, Churchwardens' Accounts, 1597-1665

St. Mary-at-the-Hill, Vestry Minutes, 1609-1752

St. Mary Colechurch, Vestry Minutes, 1612-1701 
P69/MRY8/B/005/MS00066

P69/MRY9/B/001/MS02597/001

P69/MRY9/B/007/MS02596/002

P69/MRY12/B/002/MS05714/001

P69/MRY13/B/001/MS01542/002

P69/MRY14/B/006/MS01013/001

P69/MTW/B/005/MS01016/001

P69/MIC2/B/006/MS04071/002

P69/MIC2/B/001/MS04072/001

P69/MIC3/B/009/MS01188/001

P69/MIC4/B/005/MS02895/002

P69/MIC6/B/005/MS04825/001

P69/MIC7/B/003/MS00524
St. Mary Colechurch, Churchwardens' Accounts, 1612-1700

St. Mary Magdalen Milk Street, Vestry Minutes, 1619-68

St. Mary Magdalen Milk Street, Churchwardens' Accounts, 1606/7-1666/7

St. Mary Somerset, Churchwardens' Accounts, 1614-1701

St. Mary Staining, Churchwardens' Accounts, 1644-1836

St. Mary Woolchurch Haw, Churchwardens' Accounts, 1560-1672

St. Matthew Friday Street, Churchwardens' Accounts, 1547-1678

St. Michael Bassishaw, Churchwardens' Accounts, 1617/18-1715/16

St. Michael Cornhill, Churchwardens' Accounts, 1608-1702

St. Michael Cornhill, Vestry Minutes, 1563-1697

St. Michael Crooked Lane, Churchwardens' Accounts, 1617-93

St. Michael le Querne, Churchwardens' Accounts, 1605-1717

St. Michael Queenhithe, Churchwardens' Accounts, 1625-1706

St. Michael Wood Street, Churchwardens' Accounts, 1619-1871 
P69/MIL2/B/001/MS00062/001

P69/NIC1/B/001/MS04060/001

P69/OLA2/B/004/MS04409/001

P69/OLA2/B/004/MS04409/002

P69/OLA2/B/001/MS04415/001

P69/OLA3/B/002/MS01257/001

P69/PAN/B/014/MS05018/001

P69/PAN/B/001/MS05019/001

P69/PET1/B/001/MS04165/001

P69/PET4/B/006/MS00645/002

P69/STE1/B/030/MS04456

P69/STE1/B/012/MS04457/002

P69/STE1/B/001/MS04458/001/001

P69/STE1/B/001/MS04458/001/002

P69/STE2/B/008/MS00593/004

P69/SWI/B/004/MS00559/001
St. Mildred Poultry, Vestry Minutes, 1641-1713

St. Nicholas Acons, Vestry Minutes, 1619-1738

St. Olave Old Jewry, Churchwardens' Accounts, 1586-1643

St. Olave Old Jewry, Churchwardens' Accounts, 1643-1705

St. Olave Old Jewry, Vestry Minutes, 1574-1680

St. Olave Silver Street, Churchwardens' Accounts, 1630-82

St. Pancras Soper Lane, Churchwardens' Accounts, 1616-1740

St. Pancras Soper Lane, Vestry Minutes, 1626-99

St. Peter Cornhill, Vestry Minutes, 1574-1717

St. Peter Westcheap Churchwardens' Accounts, 1601-1702

St. Stephen Coleman Street, Vellum Book

St. Stephen Coleman Street, Churchwardens' Accounts, 1586-1640

St. Stephen Coleman Street, Vestry Minutes, 1622-1693

St. Stephen Coleman Street, Vestry Minutes, 1694-1726

St. Stephen Walbrook, Churchwardens' Accounts, 1637-1748

St. Swithin London Stone, Churchwardens' Accounts, 1602-1725 


$\begin{array}{cc}\text { P69/TMS1/B/008A/MS00662/001 } & \begin{array}{c}\text { St. Thomas the Apostle, } \\ \text { Churchwardens' Accounts, } \\ 1612-1729\end{array} \\ \text { P82/AND/B/001/MS04251/001 } & \text { St. Andrew Holborn Circus, } \\ & \text { Vestry Minutes, 1642-1714 } \\ \text { P92/SAV/1898-1926, 1928, } & \text { Receipts for Maimed Souldiers, } \\ \text { 1935-1951 } & \text { St. Saviour, Southwark }\end{array}$

\section{Mercers' Company, London}

Acts of Court, 1641 to 1645

\section{Museum of London}

MS 46.78/709

Contracts for the supplying of the Army of Sir Thomas Fairfax in the year 1645

MS 46.78/673

An unsigned note relating to Lord Essex

\section{The National Archives, Kew}

E 179

SP 16

SP 19

SP 20

SP 21

SP 22

SP 23

SP 28

SP 46

SP 84
Records of the Exchequer

State Papers Domestic, Charles I

Committee for the Advance on Money

Sequestration Committee

Committee of Both Kingdoms

Committee for Plundered Ministers

Committee for Compounding with Delinquents

Commonwealth Exchequer Papers

Supplementary State Papers Domestic

State Papers Holland

\section{National Army Museum, London}

MSS 6807-53

William Levett, "The Enseigns of the Regiments in the rebellious Citty of London both of Trayned Bands and Auxiliaries," 26 September 1643 


\title{
Parliamentary Archives, Westminster
}

\author{
MAIN PAPERS HL/PO/JO/10/1/115, 117-121, 124-139, 141-148, \\ 150-158, 161-163, 165, 167-171, 176 \\ BRY/10, BRY/45, Parliamentary Records \\ $\mathrm{BRY} / 57, \mathrm{BRY} / 96$ \\ MAN/21 \\ Warrant of the Committee of Lords and \\ Commons \\ WIL/2 Papers of the Earls of Manchester

\section{Salters' Company, London}

Minute Book, 1627-84

Tower Hamlets Local History Library and Archives

W/SMH/A/1/1 Church Book

\section{Westminster Archives Centre}

E23 St. Margaret's Westminster, Churchwardens' Accounts, 1640-1

E24 St. Margaret's Westminster, Churchwardens' Accounts, 1642-3

E25 St. Margaret's Westminster, Churchwardens' Accounts, 1644-5

E2413 St. Margaret's Westminster, Vestry Minutes, 1591-1662

F2002 St. Martin-in-the-Fields, Vestry Minute Book, 1624-52

E2269 St. Clement Danes, Churchwardens' Accounts, 1627-50

\section{William Andrews Clark Memorial Library, Los Angeles}

Clark MS 1951.011 Notes from Sermons given by Richard Culverwell in St. Margaret Moyses 